\title{
Impact du lectorat visé sur la grammaire
}

Bilan du projet « Sensibilité de la grammaire anglaise au degré de spécialisation » (SGADS)

Elsa Pic et Grégory Furmaniak

\section{OpenEdition}

\section{Journals}

Édition électronique

URL : http://journals.openedition.org/asp/4199

DOI : $10.4000 /$ asp.4199

ISSN : 2108-6354

Éditeur

Groupe d'étude et de recherche en anglais de spécialité

Édition imprimée

Date de publication : 1 mars 2014

Pagination : 69-86

ISSN : 1246-8185

Référence électronique

Elsa Pic et Grégory Furmaniak, «Impact du lectorat visé sur la grammaire », ASp [En ligne], 65 | 2014, mis en ligne le 15 mars 2015, consulté le 03 novembre 2020. URL : http://journals.openedition.org/ asp/4199; DOI : https://doi.org/10.4000/asp.4199

Ce document a été généré automatiquement le 3 novembre 2020.

Tous droits réservés 


\section{Impact du lectorat visé sur la grammaire}

Bilan du projet « Sensibilité de la grammaire anglaise au degré de spécialisation » (SGADS)

\section{Elsa Pic et Grégory Furmaniak}

\section{Introduction}

1 Cet article propose une synthèse des résultats du projet « Sensibilité de la grammaire au degré de spécialisation » (SGADS), qui ont fait l'objet de plusieurs publications dans la revue ASp (Pic \& Furmaniak 2010, 2012a, 2012d; Pic et al. 2013) et ailleurs (Pic \& Furmaniak 2012b, 2012c, à paraître 1, à paraître 2 ; Furmaniak 2013). Il s'agit aussi d'apporter une réponse, sans doute provisoire mais fondée sur l'étude de nombreux phénomènes grammaticaux, à la question qui a été à l'origine du projet, à savoir : le degré de spécialisation des textes a-t-il, ceteris paribus, un impact sur l'usage des ressources grammaticales de la langue? Au-delà de cette question, cet article fournit également l'occasion de revenir sur les problèmes d'ordre méthodologique qui se sont posés à nous tout au long du projet et sur les solutions que nous y avons apportées.

2 L'étude a été menée du point de vue de ce que Douglas Biber et Susan Conrad (2009) appellent la register perspective, qui consiste à étudier la variété textuelle en mettant en relation les traits linguistiques d'un type de texte donné et ses propriétés sociopragmatiques, c'est-à-dire son contexte d'énonciation (situational characteristics) et les fonctions de communication (communicative purposes) qu'il remplit. Bien entendu, s'il peut être utile d'avoir une description a priori des propriétés des types de textes étudiés afin de rendre compte des propriétés formelles dégagées, l'analyse linguistique permet, en retour, d'affiner notre compréhension des caractéristiques socio-pragmatiques des textes (Biber et Conrad, ibidem).

3 Le projet SGADS trouve son origine dans la thématique du $31^{\mathrm{e}}$ Congrès du GERAS (Nice, mars 2010) qui proposait aux participants de s'interroger sur la notion de transversalité au sein de l'anglais de spécialité. Comme l'a rappelé la conférence inaugurale de Tony 
Bex $(2010)^{1}$, c'est d'abord la notion de genre qui vient à l'esprit quand on parle de transversalité. Au sein du discours scientifique, un genre donné (comme l'article de recherche, la communication orale, la conférence plénière, l'abstract, le résumé de thèse, etc. $)^{2}$ se définit par un certain nombre de caractéristiques structurelles, rhétoriques et linguistiques sur lesquelles la discipline a un impact suffisamment faible - quoique non négligeable (voir Fløttum et al. 2006 sur l'importance des différences disciplinaires) - pour que l'analyste puisse envisager ses différentes instanciations interdisciplinaires comme relevant du même modèle structurel et communicationnel ${ }^{3}$.

Cette transversalité s'exprime assez logiquement au sein même des genres, puisqu'un genre donné, quelle que soit la discipline, manifeste une structure rhétorique plus ou moins similaire. La structure IMRaD (Introduction, Methods/Materials, Results and Discussion), par exemple, est caractéristique de très nombreux articles de recherche empirique, avec des variations disciplinaires négligeables. Chaque division peut à son tour être découpée en divisions rhétoriques plus fines comme les moves - par exemple, au sein de l'introduction, on trouvera les sous-parties «établir un territoire de recherche » (establishing a territory) ou « créer une niche » (establishing a niche) (Swales 1990).

5 Si les notions de genre et de divisions rhétoriques sont au cœur de notre problématique, l'approche transversale de l'anglais de spécialité que nous avons retenue est fondée avant tout sur la notion de degré de spécialisation (Resche 2001) ou de public visé (intended audience - Varttala 2001). L'expert parlant de sa discipline doit en effet s'adapter à son auditoire et, au-delà des variations liées au contenu même du propos, se voit contraint d'adopter des stratégies linguistiques différentes selon qu'il s'adresse à ses pairs, au grand public, à des étudiants, à des enfants, à des amateurs éclairés, à des spécialistes d'autres disciplines, etc.

6 L'ambition du projet SGADS était donc de déterminer si, et, le cas échéant, dans quelle mesure, le degré de spécialisation des textes - quelle que soit la discipline - et, donc, le degré supposé de familiarité du lecteur avec la discipline, influencent l'utilisation par l'auteur-expert des ressources grammaticales de la langue.

7 C'est dans la mise en œuvre de cette recherche que cette transversalité rejoint celles évoquées plus haut. En effet, comment mesurer l'effet de la variable «degré de spécialisation » autrement qu'en comparant deux genres (existant dans toutes les disciplines) différenciés essentiellement par leur degré de spécialisation? Par ailleurs, comme on le verra dans la suite de l'article, la question des divisions rhétoriques s'est posée de façon aiguë dès lors qu'il s'est agi de rendre compte des différences linguistiques entre les deux genres et d'établir des liens entre phénomènes grammaticaux et fonctions textuelles.

8 Dans cet article, nous rappelons brièvement l'objet et la méthodologie du projet SGADS, avant de nous concentrer sur la présentation de ses résultats les plus marquants. Nous détaillons ensuite les hypothèses qu'il a permis de confirmer ou d'infirmer et concluons sur l'existence d'un usage grammatical propre aux articles de vulgarisation scientifique. 


\section{Corpus, méthode et résultats}

\section{1. Évolution du corpus}

9 Dans Pic \& Furmaniak (2010), nous nous appuyions sur un corpus de petite taille (100 000 mots) couvrant seulement deux disciplines: la philosophie et l'histoire. Il s'agissait à la fois d'une première exploration modeste visant à vérifier empiriquement l'existence de différences grammaticales entre le discours «hyper» spécialisé et le discours «moins » spécialisé et d'une aspiration à aborder des disciplines des sciences humaines beaucoup moins étudiées, dans le cadre de l'anglais de spécialité, que le discours du droit, des sciences exactes ou de la médecine, par exemple. Outre les limites évidentes que posait la taille du corpus, la nécessité d'étendre quantitativement et qualitativement le nombre de textes s'est très vite imposée à nous. En effet, il est rapidement apparu que les différences entre disciplines, parfois très significatives, étaient susceptibles de parasiter nos résultats. Le cas des questions (Pic et Furmaniak 2013b) est à cet égard édifiant, comme le montre le tableau 1.

Tableau 1. Fréquence des questions directes par discipline et par degré de spécialisation

\begin{tabular}{|l|l|l|}
\hline & Corpus vulgarisé & Corpus spécialisé \\
\hline Astronomie & 76 & 16 \\
\hline Economie & 110 & 60 \\
\hline Histoire & 60 & 24 \\
\hline Mathématiques appliquées & 204 & 10 \\
\hline Philosophie & 285 & 234 \\
\hline Total & 735 & 344 \\
\hline
\end{tabular}

La forte propension du philosophe à utiliser des questions à la fois en discours spécialisé et en discours vulgarisé vient quelque peu masquer le fait que la structure interrogative est globalement très marginale dans le discours universitaire (68 \% des questions dans ce sous-corpus apparaissent en philosophie). Par ailleurs, le très faible écart en philosophie (rapport de 1,2 pour 1) entre le vulgarisé et le spécialisé est à contraster avec l'écart important (rapport de 20,4 pour 1) entre les deux genres en mathématiques appliquées.

11 Afin d'atténuer au maximum ces spécificités disciplinaires et de mettre en avant la variable "degré de spécialisation», il convenait donc d'augmenter le nombre de disciplines dans le but de réduire la part de chacune d'entre elles au sein du corpus. Le corpus a ainsi compté deux, puis trois, enfin cinq disciplines (Pic \& Furmaniak 2012a, 2012c). Nous avons inclus à notre corpus des textes relevant des sciences exactes et appliquées (mathématiques et astronomie) et de l'économie. À terme, il serait souhaitable d'ouvrir encore davantage le corpus, en incluant, en particulier, une section relevant de sciences plus expérimentales. 

attention toute particulière lors de l'interprétation des résultats. En effet, et c'est là tout l'intérêt de la stratification du corpus en disciplines différentes, il convient de ne pas s'arrêter à la seule comparaison des résultats du corpus spécialisé et du corpus vulgarisé, mais de s'assurer que les différences constatées au niveau général valent au sein de chaque discipline. Si l'on reprend l'exemple des questions (tableau 1), il est significatif que leur surreprésentation en discours vulgarisé (735 occurrences contre 344 en discours spécialisé) se confirme à l'intérieur de chaque discipline (même si c'est, comme on l'a vu, dans des proportions différentes). C'est là la condition sine qua non pour pouvoir affirmer que le phénomène est lié à la variable " degré de spécialisation ».

13 À l'inverse, l'étude des modaux must, may et might (Pic et Furmaniak, 2012a ${ }^{4}$ ) montre que leur distribution, dans leur emploi épistémique, est davantage influencée par la discipline que par le degré de spécialisation.

Tableau 2. Fréquence des emplois épistémiques de must, may et might

\begin{tabular}{|l|l|l|}
\hline & Corpus vulgarisé & Corpus spécialisé \\
\hline Histoire & 60 & 90 \\
\hline Mathématiques appliquées & 109 & 41 \\
\hline Philosophie & 174 & 121 \\
\hline Total & 343 & 252 \\
\hline
\end{tabular}

Les résultats globaux (tableau 2) pourraient, à première vue, laisser penser que l'emploi des modaux épistémiques est plus spécifique au discours vulgarisé (343 occurrences contre 252 dans le corpus spécialisé). Ces résultats sont confirmés en philosophie et en mathématiques appliquées, mais pas en histoire où cette tendance est très clairement inversée. Cette donnée vient donc remettre en question la conclusion ci-dessus qui aurait pu être tirée au vu des seuls résultats totaux. Elle suggère, au contraire, que l'emploi des modaux épistémiques est davantage sensible à la discipline qu'au degré de spécialisation.

\subsection{Synthèse des phénomènes étudiés : principaux résultats}

Le tableau 3 présente les principaux résultats du projet en offrant une vue synthétique des marqueurs ou phénomènes étudiés dans chaque grand domaine grammatical et leur affinité (ou indifférence) avec l'un des deux genres. Apparaissent dans ce tableau non seulement les résultats purement quantitatifs (fondés sur la fréquence brute des formes concernées), mais aussi la «modulation » qualitative de ces résultats (fondée sur la fréquence des valeurs de ces mêmes formes). Il faut ainsi comprendre, par exemple, que le pluperfect tous emplois confondus est plus fréquent dans les articles de vulgarisation, mais que son emploi résultatif est caractéristique des articles de recherche. 
Tableau 3. Synthèse des résultats du projet SGADS

\begin{tabular}{|c|c|c|c|}
\hline & \multicolumn{3}{|c|}{ Phénomènes ou marqueurs grammaticaux } \\
\hline & Propres au SPÉCIALISÉ & Propres au VULGARISÉ & Indifférents \\
\hline \multirow{2}{*}{ 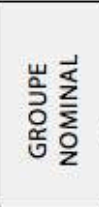 } & $\begin{array}{l}\text { - Pronoms de lère pers. } \\
\text { - I/we renvoyant à l'auteur }\end{array}$ & $\begin{array}{l}\text { - Pronoms de } 2 \text { ème pers. } \\
\text { - Pronoms de 3ème pers. sg. (s)he } \\
\text { - l/we génériques }\end{array}$ & - We inclusif \\
\hline & $\begin{array}{l}\text { Démonstratifs } \\
\rightarrow \text { This/these }\end{array}$ & & \\
\hline \multirow{2}{*}{ 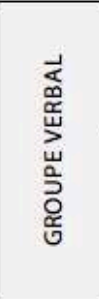 } & $\begin{array}{l}\text { - Emploi résultatif du } \\
\text { Pluperfect }\end{array}$ & $\begin{array}{l}- \text { Passé } \\
\rightarrow \text { Pluperfect } \\
\rightarrow \text { Emploi irréel du Pluperfect }\end{array}$ & $\begin{array}{l}- \text { Présent } \\
\rightarrow \text { Present perfect }\end{array}$ \\
\hline & - May, might, must radicaux & $\begin{array}{l}\text { - May, might, must épistémiques } \\
\text { - Verbes évidentiels } \\
\text { - Approximateurs } \\
\text { - Adverbes modaux } \\
\rightarrow \text { perhaps, maybe, possibly }\end{array}$ & \begin{tabular}{|l} 
- Hedges \\
- Modaux épistémiques
\end{tabular} \\
\hline \multirow{4}{*}{ 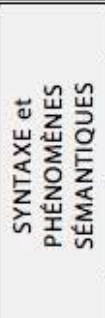 } & & & - Passif \\
\hline & - Questions polyphoniques & $\begin{array}{l}\text { - Questions } \\
\rightarrow \text { Questions rhétoriques } \\
\rightarrow \text { Questions rhéto non polyph. }\end{array}$ & \\
\hline & $\begin{array}{l}-\approx \text { Reformulateurs } " \\
\rightarrow \text { That is, namely, i.e. }\end{array}$ & & $\begin{array}{l}\text { - In other words, that is to say, I } \\
\text { mean }\end{array}$ \\
\hline & - Adjectifs de similarité & - Like (prép./conj./suffixe) & - Comparaison \\
\hline
\end{tabular}

16 C'est d'ailleurs au niveau de ces analyses plus fines que les résultats sont les plus intéressants. En effet, quand on considère uniquement une classe sémanticogrammaticale, c'est-à-dire des formes regroupées selon des critères morphosyntaxiques (par exemple, les modaux en général ou les temps grammaticaux) ou sur une base sémantique (par exemple, le hedging ${ }^{5}$ ou la comparaison), on ne trouve que peu ou pas de différences entre discours spécialisé et discours vulgarisé. Cependant, quand on se concentre sur les valeurs particulières d'une forme (par exemple, must épistémique), ou sur une sous-notion sémantico-pragmatique (par exemple, les " approximateurs $»^{6}$ ) ou encore sur une combinaison spécifique de formes (par exemple, le pronom I + un prédicat épistémique), alors apparaissent nettement les variations entre les deux genres. Comment rendre compte de ces variations ? Constater des différences, même à un degré d'analyse relativement fin, ne revient pas à expliquer les tendances observées.

$\mathrm{Au}$ cours du projet, deux hypothèses ont été successivement envisagées pour tenter de faire sens des disparités constatées entre les deux types de discours. La première provient tout à la fois des idées reçues ou des intuitions largement partagées sur la vulgarisation et de la recherche existante sur ses fonctions socio-pragmatiques. La deuxième hypothèse prend en compte la complexité et l'hétérogénéité formelles et fonctionnelles des textes en évitant des généralisations abusives sur le genre (lesquelles sont parfois fructueuses, mais peuvent aussi s'avérer inopérantes). Ces deux hypothèses sont développées ci-après afin de montrer comment elles éclairent (ou ne parviennent pas à éclairer) les résultats du tableau 3. 


\section{Les hypothèses mises à l'épreuve}

\section{1. Échec de l'hypothèse 1 : lien degré de spécialisation/ (inter)subjectivité}

Dès le départ, notre approche a été fondée sur l'analyse de notre corpus : nous nous inscrivons ainsi dans une démarche dite "corpus-driven ${ }^{7}$, au sens où l'analyse quantitative et qualitative des données du corpus visait à confirmer ou infirmer des hypothèses émises sur la base d'un examen intuitif des textes.

Ainsi, la lecture comparée des articles spécialisés, d'un côté, et des textes vulgarisés, de l'autre, donne une impression nette du style et du ton des deux genres. Des premiers se dégagent une impression de neutralité, d'objectivité, de sérieux, de formalité, une certaine aridité parfois, et des seconds le sentiment qu'on a affaire à des auteurs plus " chaleureux ", plus soucieux d'interagir avec le lecteur, n'hésitant pas à recourir à un style moins formel, voire à faire de l'humour.

À cet égard, l'appartenance des exemples (1) et (2) au corpus vulgarisé et de l'exemple (3) au corpus spécialisé apparaît comme évidente.

(1) WINTER in my part of Scotland is not the best time to start serious solar observing, but when you have been waiting some time for a new toy you tend to take any opportunity you can get. Here are some impressions of the new Lunt 50 $\mathrm{mm} \mathrm{H}$-alpha (hydrogen-alpha) etalon and B1200 blocking filter for solar viewing. (Astronomie vulgarisée)

(2) I have visited churches in Italy where there is a small hole in the roof which, like a pinhole camera, makes an image of the Sun on the floor. (Astronomie vulgarisée) (3) The Cold Dark Matter (CDM) cosmological model has recently enjoyed great success on large scales. Observational data as diverse as precision measurements of the cosmic background radiation (e.g., de Bernardis et al. 2001; Halverson et al. 2001), measurements of absorption lines in the spectra of distant quasars (e.g., Efstathiou, Schaye \& Theuns 2000), measurements of the abundances of deuterium, and other light elements (e.g., Burles, Nollett \& Turner 2001), surveys of the positions in redshift space of hundreds of thousands of galaxies (e.g., Peacock et al. 2001) and measurements of the brightnesses of distant supernovae (e.g., Reiss et al. 1998; Perlmutter et al. 1999) all point to a single cosmological model. (Astronomie spécialisée)

21 Familiers des notions de dialogisme et de polyphonie, ainsi que des travaux sur des phénomènes comme la modalité, le hedging ou l'emploi des pronoms personnels dans les articles de recherche, nous avions bien sûr conscience que ce dernier n'était pas un genre purement objectif et monologal et que l'analyse des données montrerait qu'il n'est pas exempt de traces de subjectivité et d'interaction. Il nous semblait cependant que les observations intuitives mentionnées plus haut se reflèteraient dans l'examen systématique de l'usage grammatical propre à chaque genre.

En effet, un certain nombre de résultats confirment ces intuitions, en particulier la dimension interpersonnelle beaucoup plus marquée en discours vulgarisé qu'en discours spécialisé. La fréquence largement supérieure du pronom you en discours vulgarisé (voir tableau 4, tiré de Pic \& Furmaniak 2012a) en est un premier indice. 
Tableau 4. Fréquence du pronom you

\begin{tabular}{|l|l|l|}
\hline & Corpus vulgarisé & Corpus spécialisé \\
\hline Histoire & 28 & 0 \\
\hline Mathématiques appliquées & 606 & 12 \\
\hline Philosophie & 224 & 120 \\
\hline Total & 858 & 132 \\
\hline
\end{tabular}

Il en va de même des questions (voir tableau 1), marques d'interaction par excellence (Hyland 2002). Même si, dans les deux genres, les questions ne sont pas prototypiques au sens où elles ne sollicitent pas une réponse verbalisée de la part du lecteur, leur emploi dans de tels contextes (Thompson et Thetela 1995) est un moyen d'impliquer ce dernier dans l'événement communicationnel, en lui attribuant un rôle actif, typiquement celui du questionneur, comme en (4), et, en particulier dans les questions rhétoriques telles que (5), celui du " répondeur ».

(4) Similarly, claims widely recognised as unrealistic are a feature of seemingly all mathematical deductivist endeavour in modern economics (...). Why should this be? And specifically, why do I suppose that the emphasis on formalistic modelling is the problem? My answer, simply put, can be expressed in the following three propositions. (Économie spécialisée)

(5) This research might be slightly offensive to pasta lovers - who wants to eat short spaghetti? (Mathématiques appliquées vulgarisées)

Bien sûr, l'auteur reste maître du jeu. C'est lui qui attribue les rôles interactionnels et qui impose le contenu des questions et des réponses censées émaner du lecteur. En (4), l'auteur fait poser au lecteur les questions qui lui permettent de faire avancer son argumentation, tandis qu'en (5), la question rhétorique « force » la réponse No one does. Leur rôle interpersonnel est cependant essentiel, puisque même si la question répond à une stratégie discursive de l'auteur, elle reconnaît explicitement l'existence du lecteur et anticipe ses besoins.

Malgré ces résultats, distinguer les deux genres en termes de subjectivité et d'intersubjectivité ne s'est pas toujours avéré possible. D'abord, comme l'a montré Jan Nuyts (2001), ces concepts sont problématiques et sont loin d'être définis de manière consensuelle. La surreprésentation, en discours spécialisé, du pronom we référant à l'auteur (ou aux auteurs, Pic \& Furmaniak [2012c]) serait interprétée par certains (Nuyts, ibidem) comme une forte trace de subjectivité, alors qu'elle serait vue par un cognitiviste comme Ronald Langacker (2002) comme une marque d'objectivisation de l'auteur. En effet, s'il est admis que les constructions avec extraposition du type it is possible that expriment une modalité plus objective qu'un modal comme may (Lyons 1977, Nuyts 2001, Huddleston \& Pullum 2002, Ressano 2004), on peut légitiment hésiter quand il s'agit de classer des formes telles que may, I think ou maybe sur une échelle de subjectivité.

Par ailleurs, l'opposition subjectif/objectif n'est pas pertinente pour un certain nombre de phénomènes grammaticaux, par exemple, la construction clivée, l'emploi de 
marqueurs paraphrastiques de reformulation (Pic et al. 2013), de démonstratifs ou du temps grammatical passé (Pic \& Furmaniak 2012a).

Enfin, certains résultats semblent contredire l'hypothèse. Quantitativement, les hedges sont aussi nombreux en discours spécialisé qu'en discours vulgarisé (Pic \& Furmaniak 2012d), tandis que les pronoms de première personne sont nettement plus nombreux dans les articles de recherche (3 894 contre 2938 en discours vulgarisé8).

Ces différentes études de cas nous ont montré que vouloir ramener la différence entre articles de recherche et articles vulgarisés à des dichotomies telles que objectivité/ subjectivité, personnel/impersonnel ou distance/proximité revenait à ignorer la richesse des textes (qui sont connus pour être des unités hétérogènes tant au niveau formel qu'au niveau fonctionnel). Elles nous ont aussi montré qu'il était erroné de penser que les fonctions socio-pragmatiques des deux genres (si tant est qu'elles puissent être clairement identifiées) se traduiraient directement dans les formes grammaticales utilisées. Il paraît clair, en effet, que le style plus « personnel » et moins formel des articles vulgarisés ne se reflète pas aussi directement que nous l'avions d'abord pensé dans les phénomènes grammaticaux. Les liens entre propriétés linguistiques et genres sont plus complexes et plus diffus que nous ne l'avions envisagé.

\subsection{Validité de l'hypothèse 2 : les modes rhétoriques comme fait explicateur}

29 Face aux résultats insuffisamment fructueux auxquels a mené cette première hypothèse, nous avons opté pour une autre stratégie consistant à s'appuyer sur les divisions rhétoriques des textes. Les linguistes travaillant dans le champ des langues de spécialité limitent souvent ainsi leurs analyses à certaines parties des textes scientifiques ou, en tout cas, distinguent soigneusement les parties manifestant des propriétés formelles et fonctionnelles propres. Les analyses portant sur les articles de recherche se sont ainsi largement appuyées sur la structure IMRaD, mode de structuration des articles communément utilisé, en particulier dans les sciences exactes et/ou expérimentales. La nécessité de fragmenter les genres en unités discursives plus fondamentales est ainsi bien connue. Toutefois un problème se pose dès que l'on souhaite comparer les propriétés de genres différents. Si deux types de textes possèdent des structures rhétoriques dissemblables, comparer des parties ou sousparties dissimilaires n'a pas grand sens. Dans sa thèse consacrée au hedging dans les articles spécialisés et vulgarisés, Teppo Varttala (2001), confronté aux mêmes problématiques, a proposé une division rhétorique commune aux deux genres, en trois parties: introduction, corps du texte et conclusion. Si cette solution traduit une heureuse prise en compte du problème, elle n'est pas satisfaisante car la partie « corps du texte » ne manifeste aucune unité linguistique et fonctionnelle, réduisant ainsi à néant l'intérêt d'une approche rhétorique.

30 La solution qui s'est imposée à nous a été de recourir à un type de division rhétorique classique (Narration, Description, Argumentation, etc..$^{9}$ ) en faisant la synthèse de deux courants de pensée qui se sont réappropriées ces catégories et en ont proposé une nouvelle théorisation. Ces unités constitutives des genres (Adam 1992) ne sont pas, contrairement aux divisions IMRaD ou aux moves, spécifiques à un genre particulier, ni d'ailleurs au discours scientifique. Même si - et parce que - tous les modes rhétoriques ne sont pas mobilisés dans tous les genres textuels, ils constituent un outil d'analyse 
capable de comparer la structure rhétorique de genres variés. Le fait divers, par exemple, se distingue par le recours quasi-exclusif à la Narration, mode qui est totalement absent du genre de l'abstract.

S'ils conservent peu ou prou les mêmes catégories et s'entendent sur un certain nombre de critères définitoires, les deux mouvements - l'école française représentée par Jean-Blaise Grize (1990) ou Jean-Michel Adam (1992) et l'école anglo-saxonne représentée par Carlota Smith (2003) dans la lignée de Cleanth Brooks et Robert P. Warren (1979) - se distinguent par leur projet et donc, par leur approche, plutôt fonctionnelle pour le premier, plutôt formelle pour le second, de ce que nous appellerons désormais les modes rhétoriques. J.-M. Adam (1992) se propose de dégager des types de séquences et des modes d'organisation séquentielle susceptibles de rendre compte de la diversité des genres. La grammaire joue un rôle secondaire dans son modèle (Adam 1992 : 39). À l'inverse, le souci de C. Smith (2003) est d'identifier des liens entre grammaire et discours. Elle part ainsi de l'observation que certaines configurations aspectuelles sont propres à certains modes rhétoriques. La Narration, par exemple, se caractérise grammaticalement par la présence de procès (événements ou états) spécifiques localisés dans le temps les uns par rapports aux autres. Ce sont donc, dans cette approche, les liens entre micro-fonctions et méso-fonctions qui sont mis en exergue, les macro-fonctions des genres passant quelque peu au second plan $^{10}$.

Nous ne revenons pas ici sur la description détaillée des huit différents modes rhétoriques que nous avons déjà eu l'occasion de présenter ailleurs (Pic \& Furmaniak 2012d) : Narration, Description, Report, Information, Argumentation, Métadiscours, Instruction, Dialogue. En revanche, au cours de l'avancement du projet, nous avons dû ajouter un neuvième mode, la Description d'actions. Il s'agit d'un mode présenté par J.M. Adam (ibid.) comme un cas particulier de la Description qui décrit comment, à un moment spécifique, une entité est/a été/sera faite. Comme la Narration, la Description d'actions utilise des procès dynamiques, mais elle s'en distingue par l'absence des phases traditionnellement attribuées à la Narration (complication, résolution et chute). Dans notre corpus, les Descriptions d'actions apparaissent essentiellement dans la partie méthodologique des articles de recherche (voir exemple 6), où l'auteur retrace les différentes étapes d'une procédure.

(6) The X-ray surface brightness is calculated from the known electron density and temperature distribution of a simulated cluster by using equation (19). The resulting surface brightness map is then multiplied by the count rate conversion factor of a typical instrument (the EPIC camera on XMM-Newton), which is assumed to be constant across the $0.5-2 \mathrm{keV}$ energy band. We use a typical neutral hydrogen column density of $10^{20} \mathrm{~cm}^{-2}$ and an integration time equal to $10 \mathrm{ks}$. The

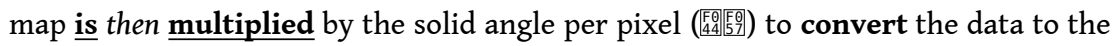
expected counts per pixel. (Astronomie spécialisée)

L'ordre linéaire du texte retraçant l'ordre chronologique des étapes de la procédure, on trouve des prépositions, des conjonctions ou des adverbes temporels (en italiques) et des nombres ordinaux. Les verbes sont essentiellement des verbes dynamiques (en gras) qui sont assez fréquemment à la voix passive (soulignés).

Pragmatiquement, ce mode est assez proche de l'Instruction, au point que J.-M. Adam (ibid., 95) choisit de ne pas les distinguer. Dans les deux cas, on a affaire à des types procéduraux décrivant les étapes intermédiaires conduisant à un résultat. On peut toutefois différencier les deux modes grâce aux notions de "valeur patente » et de «valeur latente » (Kerbrat-Orecchioni 1986: 66). Si l'une des finalités de la Description 
d'actions est de permettre à la communauté scientifique de reproduire l'expérimentation décrite (fonction instructionnelle latente), la fonction patente d'un tel passage est avant tout constative. Une glose possible est «voilà comment nous avons procédé ".

Nous avons également déjà démontré la pertinence de ces modes rhétoriques pour la caractérisation des deux types de discours (Pic \& Furmaniak 2012d, à paraître 1). La figure 1 présente la distribution, réactualisée en fin de projet, des neuf différents modes dans les deux genres. Il reste flagrant que leur répartition est très différente en discours spécialisé et en discours vulgarisé : les articles de recherche ne recourent principalement qu'à trois modes (Argumentation, Information, Métadiscours) et à deux de façon marginale (Narration et Description d'actions), alors que les articles de vulgarisation sont caractérisés par l'utilisation, plus également répartie, de presque tous les modes rhétoriques.

Figure . Distribution des modes rhétoriques en discours spécialisé et vulgarisé

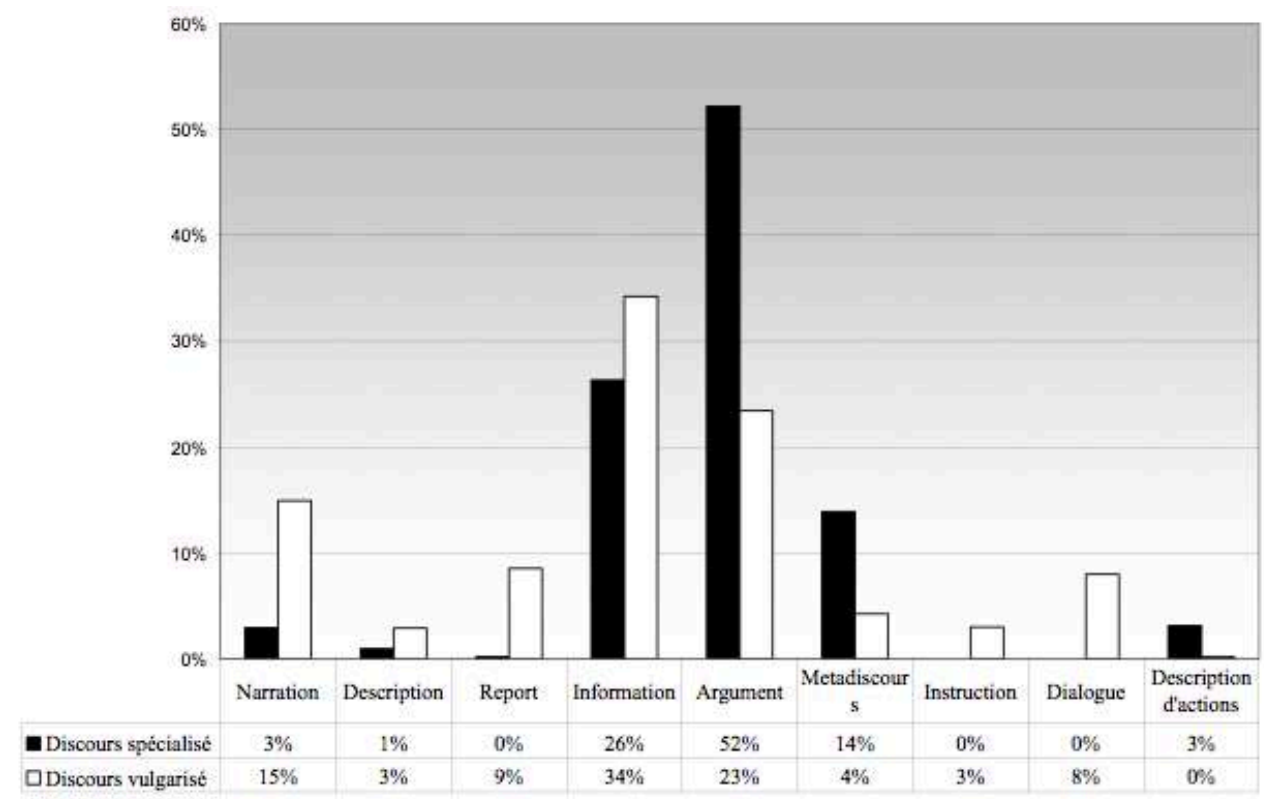

Il semble donc opportun et légitime de considérer les modes rhétoriques comme l'interface adéquate pour éclairer les liens entre textes et grammaire dans notre corpus. Nous illustrons maintenant leur efficacité à rendre compte des résultats obtenus.

\section{Les modes rhétoriques comme interface adéquate entre genre et grammaire}

\subsection{L'exemple de la Narration}

37 La Narration est associée à certaines propriétés formelles. Même si J.-M. Adam (ibid., 71) est dubitatif quant à la possibilité "d'énumérer des marques de surface », les travaux de C. Smith (ibid.) ont montré qu'établir un lien entre grammaire et modes rhétoriques était possible à condition qu'on ne se limite pas aux formes elles-mêmes, 
mais à des valeurs ou opérations sémantiques plus abstraites telles que l'aspect (lexical et grammatical), le type de repérage, les circonstants, les références aux personnes, etc. procès bornés : le prétérit simple et le pluperfect. Ces formes sont plus nombreuses en discours vulgarisé (8 160 occurrences) qu'en discours spécialisé (6 209 occurrences). À nouveau, en connaissant la propension du mode narratif à utiliser ces temps et la proportion plus importante de la Narration en discours vulgarisé, ces résultats confirment l'hypothèse de départ.

\subsection{L'exemple des adverbes épistémiques perhaps et maybe}

Le tableau 3 faisait apparaitre les adverbes épistémiques perhaps, maybe et possibly comme caractéristiques du discours vulgarisé ${ }^{11}$. Concentrons-nous sur les deux premiers. Leur fréquence dans les articles de vulgarisation est presque deux fois supérieure à leur fréquence dans les articles de recherche : 160 occurrences contre 92, si l'on s'en tient à leurs deux valeurs dominantes en corpus, la conjecture (illustrée par [7]) et la prudence oratoire (tentative use, illustrée par [8]). La conjecture revient à la formulation d'une hypothèse, la prudence oratoire consiste à nuancer une affirmation.

(7) Perhaps these are the waste gases given off by hardy bacteria living deep beneath the freezing Martian surface. (Mathématiques appliquées vulgarisées)

(8) The claim that God is a person is, perhaps, one of the most fundamental claims which believers make about God. (Philosophie vulgarisée)

43 Comment expliquer la prédominance de perhaps et maybe en discours vulgarisé ? Il nous semble que quatre pistes sont envisageables et surtout, non mutuellement exclusives. Les trois premières concernent les marqueurs eux-mêmes (leur portée, leur position, 
leur composante évidentielle) et la quatrième, le mode rhétorique avec lequel ces deux adverbes ainsi caractérisés ont des affinités patentes (le mode explicatif).

La première explication à la surreprésentation de perhaps et maybe dans le discours vulgarisé tient à la portée de ces adverbes. En effet, ils peuvent porter soit sur un fait (c'est le cas en [7]), soit sur une évaluation (comme en [8]) ${ }^{12}$. Or, dans les articles de recherche, ces adverbes qualifient très majoritairement des évaluations, comme si l'incertitude de l'auteur portait davantage sur l'interprétation des faits que sur les faits eux-mêmes. Il n'existe pas de telle limitation à la portée (et donc à l'utilisation) de perhaps et maybe dans les articles de vulgarisation, où ils modulent presque indifféremment faits et évaluations.

La deuxième piste réside dans la position de ces adverbes en corpus, qui est majoritairement la position initiale. Au niveau de la gestion de l'information, cette position met au premier plan l'incertitude de l'auteur. Comparons (9) et (9') :

(9) Maybe lightning struck some chemicals dumped in a swamp and triggered spontaneous cellular activity that issued in me. (Philosophie vulgarisée)

(9') Lightning may have struck some chemicals dumped in a swamp and triggered spontaneous cellular activity that issued in me.

En (9) maybe en position initiale focalise l'incertitude, laquelle passe au second plan en $\left(9^{\prime}\right)$, grâce à l'utilisation du modal may, nécessairement en position médiane. L'auteur d'articles de recherche favorisera sans doute cette dernière solution, qui attire moins l'attention sur ses doutes que ne le ferait un adverbe en position initiale.

Une autre façon, non exclusive, d'expliquer le phénomène est liée à la notion d'évidentialité. Il est en effet difficile, selon nous, de dissocier modalité épistémique et évidentialité (Frank Palmer 2001 réunit d'ailleurs les deux catégories). On peut donc dire, à la suite de Gordon Tucker (2002: 197) que la différence entre des formes exprimant un degré de probabilité similaire peut s'analyser en termes de la qualité sous-entendue des preuves qui sous-tendent le jugement modal. Tandis que perhaps et maybe signalent un jugement " considérablement spéculatif et prudent ${ }^{13}$ » (ibid.: 198), le modal may, dans son emploi épistémique, implique plutôt l'existence d'indices susceptibles d'étayer le jugement de probabilité ${ }^{14}$. L'exemple (10), qui contient une occurrence de chacune des deux formes, est à cet égard révélateur.

(10) (...) the diary suggests that landladies who lived alone, or with a female relative, may have had access to similar amounts of space as their lodgers and were perhaps more willing to bend to a lodger's demands than a landlord or married landlady. Sadly, no diaries of female lodgers in this period have come to light... (Histoire spécialisée)

La première conjecture, marquée par le modal et portant sur un fait, s'appuie sur une source historique tangible (the diary suggests). La seconde, exprimée par l'adverbe, est plus spéculative. Elle porte sur une évaluation, une volition pour être précis (be more willing), qui, en tant que telle, est nécessairement moins vérifiable qu'une réalité physique. Par ailleurs, la phrase suivante (en italiques), laisse entendre que les sources qui auraient pu permettre d'étayer cette hypothèse n'existent pas.

La dernière explication permettant de rendre compte de la fréquence plus élevée de perhaps/maybe en discours vulgarisé se situe au niveau discursif et fait appel aux modes rhétoriques. Les adverbes apparaissent en effet régulièrement dans des passages de type explicatif. L'Explication est ici considérée comme une sous-catégorie du mode de l'Information. Elle se distingue du sous-mode non marqué (que nous appellerons Exposition) par sa structure et par l'asymétrie épistémique auteur/lecteur qui la sous- 
tend. On considère, à la suite de J.-B. Grize (1990), J.-M. Adam (1992) et Sophie Moirand (1999), que le passage explicatif répond à la structure prototypique suivante : (i) renvoi à une réalité établie, (ii) question de type « Pourquoi ?", «Comment ça marche? » ou "Qu'est-ce que ça veut dire? " à propos de cette réalité, (iii) explication proprement dite proposée par l'auteur et (iv) évaluation et/ou conclusion. On retrouve cette structure dans l'exemple (11), avec un léger écart par rapport au prototype.

(11) Eliot's lines, Carey maintains, 'escape sense'. He points out that there is much scholarly debate about what the three leopards represent; Are they a reference to I Kings 19? Is there an allusion to Jakob Grimm's story [...], and/or to the portrayal of leopards to be found in medieval literature? Is there significance in the order in which Eliot's leopards devour their victim? 'We cannot tell. Nor do we know how serious Eliot is being.' Perhaps the leopards are metaphorical; perhaps they are to be viewed as heraldic emblems. We have also to decide on the identity of the 'Lady' being addressed, though 'there is nothing in the rest of the poem to clarify it. So we must do our best to fabricate some sort of identity for her too.'

50 Le passage commence par renvoyer à une réalité existante (un poème d'Eliot) et s'interroge (indirectement) sur sa signification (escape sense, much scholarly debate). Les questions fermées qui suivent apparaissent comme autant de tentatives d'explication que l'auteur - et c'est là que l'on sort de la structure prototypique n'assume pas complètement. L'échec du processus explicatif est alors explicitement signifié par We cannot tell. Les énoncés introduits par perhaps relancent, en quelque sorte, ce processus, avec de "nouvelles» explications encore plus hypothétiques que les précédentes, parce qu'apparemment non fondées (voir point précédent). Cette clôture sur une note hautement spéculative semble toutefois préférable (et est en tout cas ici préférée) à une absence d'explication. Finir sur We cannot tell ne serait en effet pas pragmatiquement satisfaisant (au sens où la fonction du passage explicatif ne serait pas accomplie) et qu'une telle explication était tout de même préférable à un constat d'échec.

\section{Conclusion}

51 Le projet SGADS s'était donné pour but de répondre à la question: «la grammaire anglaise est-elle sensible au degré de spécialisation des textes ?». Au terme de ce projet, il semble que l'on puisse répondre à cette question par l'affirmative, puisque nous avons mis au jour de nettes différences grammaticales, à la fois quantitatives et qualitatives, qui transcendent les cinq disciplines considérées.

Il faut bien sûr nuancer: sans doute toute la grammaire n'est-elle pas sensible prioritairement au degré de spécialisation, et certains phénomènes restent surtout liés à une discipline - c'est le cas, semble-t-il, des modaux épistémiques pour lesquels les différences entre les disciplines l'emportent sur la variable « degré de spécialisation ».

Nous espérons avoir montré qu'il est de toute façon très complexe d'établir des corrélations car de multiples facteurs entrent en jeu, en particulier les différentes fonctions d'un même marqueur, mais que le recours aux modes rhétoriques permet de mieux comprendre les variations grammaticales entre les deux genres.

Si ce projet se termine institutionnellement, il n'en reste pas moins de nombreux autres points à explorer: nous n'avons pas épuisé les phénomènes grammaticaux à étudier et le lexique reste aussi à analyser. Les retombées didactiques sont également riches, en particulier pour l'enseignement de l'anglais dans le secteur LANSAD. Enfin 
une approche contrastive français/anglais des deux genres apporterait un éclairage intéressant sur l'influence du facteur culturel dans le processus de vulgarisation.

\section{BIBLIOGRAPHIE}

Adam, Jean-Michel. 1992. Les textes : types et prototypes. Paris : Nathan.

Benveniste, émile.1966. Problèmes de linguistique générale, 1. Paris : Gallimard.

Alonso-Almeida, Francisco \& Laura Cruz-García. 2011. “The value of may as an evidential and epistemic marker in English medical abstracts". Studia Anglica Posnaniensia 46/3, 59-73.

Bex, Tony. 2010. “What are genres and should we teach them?". ASp 58, 5-18.

Biber, Douglas \& Susan Conrad. 2009. Register, Genre, and Style. Cambridge : Cambridge University Press.

Biber, Douglas et alii. 1999. Longman Grammar of Spoken and Written English. Londres : Longman.

Bordet, Geneviève. 2011. "Étude contrastive de résumés de thèse dans une perspective d'analyse de genre ». Thèse de doctorat, Université Paris Diderot.

Brooks, Cleanth \& Robert Penn Warren. 1979. Modern Rhetoric. New York : Harcourt Brace Jovanovich.

Dijk, Teun A van. 1998. “Opinions and ideologies in the press”. In Bell, A. \& P. Garrett (eds.), Approaches to Media Discourse. Blackwell : Oxford, 1-63.

Fløttum, Kjersti, Trine Dahl \& Torodd Kinn. 2006. Academic Voices - across languages and disciplines. Amsterdam : John Benjamins.

Furmaniak, Grégory. 2013. «Vers une grammaire pédagogique de l'anglais pour le LANSAD ». Études en Didactique des Langues 20, 13-27.

Grize, Jean-Blaise. 1990. Logique et langage. Paris : Ophrys.

Huddleston, Rodney \& Geoffrey K. Pullum. 2002. The Cambridge Grammar of the English Language. Cambridge : Cambridge University Press.

Hyland, Ken. 2002. "What do they mean? Questions in academic writing”. Text 22/4, 529-557.

Kerbrat-Orecchioni, Catherine. 1986. L'implicite. Paris : Armand Colin.

Langacker, Ronald. 2002. Concept, Image and Symbol: The cognitive basis of grammar. New York: Mouton de Gruyter.

Lyons, John. 1977. Semantics 2. Cambridge : Cambridge University Press.

Moirand, Sophie. 1999. « L'explication ». In Beacco, J.-C. (dir.), L'astronomie dans les médias. Paris : Presses de la Sorbonne Nouvelle, 141-167.

Nuyts, Jan. 2001. Epistemic Modality, Language, and Conceptualization. Philadelphie, PA : John Benjamins.

Palmer, Frank. 2001. Mood and Modality [2nd edition]. Cambridge : Cambridge University Press. 
Pic, Elsa \& Grégory Furmaniak. 2010. « Grammaire et degré de spécialisation ». ASp 58, 39-55

Pic, Elsa \& Grégory Furmaniak. 2012a. « De la langue spécialisée à la langue vulgarisée: approche grammaticale ». ASp 61, 39-54.

Pic, Elsa \& Grégory Furmaniak. 2012b. "A study of epistemic modality in academic and popularised discourse: The case of possibility adverbs Perhaps, Maybe and Possibly". Revista de Lenguas para Fines Específicos 18, 11-44.

Pic, Elsa \& Grégory Furmaniak. 2012c. « Le degré de spécialisation comme facteur de variation de la représentation du JE ». In Frath, P. et alii (dir.), Res-per-nomen III : La référence, la conscience et le sujet énonciateur / Reference, Consciousness and the Speaking Subject. Reims : Epure, 193-218.

Pic, Elsa \& Grégory Furmaniak. 2012d. « Les modes de discours, interface entre texte et grammaire en langue de spécialité ». ASp 62, 25-44.

Pic, Elsa \& Grégory Furmaniak. 2013a. « Les modes rhétoriques comme lien entre genre textuel et grammaire : le cas de la Narration dans les articles de vulgarisation ». Actes du colloque « Usages et fonctions de la rhétorique ». Bruxelles, GRAL, mai 2013. Publication en ligne : <http:// rheto13.ulb.ac.be/forum>. Consulté le 30 janvier 2014.

Pic, Elsa \& Grégory Furmaniak. 2013b. "Questions and facework: Research articles and popular science articles compared". Communication au Sosnowiec Symposium on Communication across Cultures, avril 2013

Pic, Elsa \& Grégory Furmaniak. À paraître 1. « Quelle grammaire pour l'étudiant en voie de spécialisation? ». Cahiers de l'APLIUT.

Pic, Elsa \& Grégory Furmaniak. À paraître 2. "On the use of modalized wh-interrogatives to question certainty in research articles and popular science articles”. Ouvrage collectif. John Benjamins.

Pic, Elsa, Grégory Furmaniak \& Vincent Hugou. 2013. «Étude comparative de quelques marqueurs de reformulation paraphrastique dans les articles de recherche et les articles de vulgarisation ». ASp 63, 75-92.

Resche, Catherine. 2001. «Réflexions sur la frontière entre langue générale et langue spécialisée ». In Mémet, M. \& M. Petit (dir.), L'anglais de spécialité en France. Bordeaux : GERAS Éditeur, 37-46.

Ressano, Norma Susana. 2004. "Modality and modal responsibility in research articles in English". In Facchinetti, R. \& F. Palmer (eds.), English Modality in Perspective: Genre Analysis and Contrastive Studies. Frankfort : Peter Lang, 101-118.

Smith, Carlota. 2003. Modes of Discourse. The local Structure of Texts. Cambridge : Cambridge University Press.

Thompson, Geoff \& Puleng Thetela. 1995. "The sound of hand clapping: The management of interaction in written discourse". Text 5/1, 103-127.

Tognini-Bonelli, Elena. 2001. Corpus Linguistics at Work. Amsterdam et Philadelphie : John Benjamins.

Tucker, Gordon. 2001. "Possibly alternative modality”. Functions of Language 8/2, 183-216.

Varttala, Teppo. 2001. "Hedging in scientifically oriented discourse: Exploring variation according to discipline and intended audience". Doctoral dissertation. University of Tampere. 


\section{NOTES}

1. Une sélection des communications présentées à ce congrès a fait l'objet d'une publication dans le numéro 58 d'ASp.

2. La notion de genre dépasse bien entendu le domaine du discours scientifique (on peut citer, à titre d'exemples, la recette de cuisine, l'éditorial, l'article de fait divers, etc.). C'est toutefois au sein de celui-ci que sa transversalité est la plus flagrante.

3. Comme le rappellent fort justement D. Biber et S. Conrad (2009), il n'y a pas, dans l'analyse des genres ou des registres, un «bon » niveau de spécificité. En fonction des objectifs de l'étude, le niveau de granularité peut être très faible (c'est le cas, par exemple, dans Biber et al. 1999 qui compare trois genres très généraux : la fiction, le discours universitaire et la conversation) ou très fin (voir par exemple l'étude de Geneviève Bordet [2011] sur les résumés de thèse).

4. L'analyse a été menée sur le corpus à une étape intermédiaire de sa constitution, à savoir sur seulement trois disciplines. Cette limite n'a aucune incidence sur la présente démonstration.

5. Sont considérés comme hedges les marqueurs venant nuancer une assertion. Selon Françoise Salager-Meyer (1994), le hedge remplit deux fonctions principales: avancer un argument prudemment (précaution oratoire) ou marquer une authentique incertitude.

6. Les « approximateurs » sont une catégorie de marqueurs de hedging (par exemple, les adverbes around, about, roughly, approximatively...).

7. On doit à Elena Tognini-Bonelli (2001) l'utile distinction entre corpus-based studies et corpusdriven studies.

8. Résultats sur trois disciplines (Pic \& Furmaniak 2012c).

9. Les modes rhétoriques sont signalés par une majuscule.

10. Les micro-, méso- et macro-fonctions sont des fonctions sémantiques et/ou pragmatiques associées à des unités textuelles de tailles et de niveaux différents : la phrase (pour ce qui est des micro-fonctions), le passage rhétorique (pour les méso-fonctions) et le texte, ici, l'article (pour les macro-fonctions).

11. Pour une étude approfondie de ces trois marqueurs, voir Pic \& Furmaniak (2012b).

12. Sur l'opposition fait/évaluation, voir van Dijk (1998 : 29). Schématiquement, les faits peuvent être définis comme des procès relevant de la réalité physique, tandis que les évaluations sont des procès relevant de la réalité mentale.

13. Notre traduction.

14. Nous étendons ici l'analyse de may proposée par Francisco Alonso-Almeida et Laura CruzGarcía (2011) à tous les emplois épistémiques du modal. Pour une analyse plus développée, voir Pic \& Furmaniak (2012b)

\section{RÉSUMÉS}

Cet article présente une synthèse des principaux résultats d'un projet de recherche de deux ans consacré à la sensibilité de l'usage grammatical à la variable " public visé ». Après être revenus sur les objectifs et la méthodologie du projet, les auteurs montrent que l'utilisation des ressources grammaticales de la langue par les experts varie de façon significative, à la fois quantitativement et qualitativement, en fonction du public visé. Les résultats suggèrent toutefois que certains marqueurs ou catégories sont davantage sensibles à la discipline qu'au degré de spécialisation. L'article revient ensuite sur les hypothèses émises au cours du projet pour rendre 
compte des résultats observés. Il apparaît que le lien entre les traits [+ vulgarisé] et [+ subjectif] était trop simpliste pour pouvoir rendre compte de la complexité des phénomènes. Le passage par l'interface pragmatico-formelle que constituent les modes rhétoriques s'avère plus opératoire.

This paper summarizes the main findings of a two-year research project on the impact of intended audience on grammatical usage. After describing the main goals of the project and the methodology used, we show that the way experts use the grammatical resources of the language is significantly influenced - both quantitatively and qualitatively - by the intended audience variable. The data suggest, however, that the use of some forms or semantic categories is more strongly affected by the discipline variable. The working hypotheses are then assessed and we suggest that the assumption that 'the less specialized a text is, the more subjective it is' amounts to an over-simplification of the data. Instead, we surmise that a rhetorical analysis of the genres under scrutiny provides a more fruitful way of accounting for the linguistic differences induced by the intended audience variable.

\section{INDEX}

Mots-clés : article de recherche, public (visé), variation grammaticale, vulgarisation

Keywords : audience (intended), grammatical variation, popularization, research article

\section{AUTEURS}

\section{ELSA PIC}

Agrégée d'anglais, Elsa Pic est maître de conférences en linguistique anglaise à l'Université Sorbonne Nouvelle - Paris 3. Ses recherches portent sur la terminologie, l'anglais juridique et les spécificités linguistiques des langues de spécialité. elsa.pic@univ-paris3.fr

\section{GRÉGORY FURMANIAK}

Agrégé d'anglais, Grégory Furmaniak est maître de conférences en linguistique anglaise à l'Université Sorbonne Nouvelle - Paris 3. Il a une longue expérience de l'enseignement en secteur LANSAD. Ses recherches portent sur l'aspect et la modalité en synchronie et en diachronie, ainsi que sur l'anglais de spécialité. gregory.furmaniak@univ-paris3.fr 